\title{
Kaon decays shedding light on massless dark photons
}

\author{
Jhih-Ying Su ${ }^{1}$, Jusak Tandean ${ }^{1,2, a} \mathbb{C}_{\mathbb{C}}$ \\ ${ }^{1}$ Department of Physics, National Taiwan University, Taipei 106, Taiwan \\ 2 Physics Division, National Center for Theoretical Sciences, Hsinchu 300, Taiwan
}

Received: 15 June 2020 / Accepted: 9 August 2020 / Published online: 5 September 2020

(C) The Author(s) 2020

\begin{abstract}
We explore kaon decays with missing energy carried away by a massless dark photon, $\bar{\gamma}$, assumed to have flavor-changing dipole-type couplings to the $d$ and $s$ quarks. We consider in particular the neutral-kaon modes $K_{L} \rightarrow \gamma \bar{\gamma}$ and $K_{L} \rightarrow \pi^{0} \gamma \bar{\gamma}$ and their $K_{S}$ counterparts, as well as the charged-kaon channel $K^{+} \rightarrow \pi^{+} \gamma \bar{\gamma}$, each of which also has an ordinary photon, $\gamma$, in the final state. In addition, we look at $K_{L, S} \rightarrow \pi^{+} \pi^{-} \bar{\gamma}$ and $K^{+} \rightarrow \pi^{+} \pi^{0} \bar{\gamma}$. Interestingly, the same $d s \bar{\gamma}$ interactions give rise to the flavorchanging two-body decays of hyperons with missing energy and are subject to model-independent constraints that can be inferred from the existing hyperon data. Taking this into account, we obtain branching fractions $\mathcal{B}\left(K_{L} \rightarrow \gamma \bar{\gamma}\right)$ and $\mathcal{B}\left(K_{L} \rightarrow \pi^{0} \gamma \bar{\gamma}\right)$ which can be as high as $10^{-3}$ and $10^{-6}$, respectively, one or both of which may be within the sensitivity reach of the KOTO experiment. Furthermore, we find that $\mathcal{B}\left(K^{+} \rightarrow \pi^{+} \gamma \bar{\gamma}\right)$ and $\mathcal{B}\left(K^{+} \rightarrow \pi^{+} \pi^{0} \bar{\gamma}\right)$ are allowed to be maximally of order $10^{-6}$ as well, which may be probed by NA62. Complementarily, the hyperon modes can have rates which are potentially accessible by BESIII. Thus, these ongoing experiments could soon be able to offer significant tests on the existence of the massless dark photon.
\end{abstract}

\section{Introduction}

Over the past few decades various phenomenological considerations have motivated the introduction of the so-called dark photon, a spin-one boson associated with a new Abelian gauge symmetry, $\mathrm{U}(1)_{D}$, under which all the fields of the standard model (SM) are singlets [1-25]. The dark photon may be massive or massless, depending on whether $\mathrm{U}(1)_{D}$ is spontaneously broken or stays unbroken, respectively. The massive one, often symbolized by $A^{\prime}$, can interact directly with SM fermions through a renormalizable operator, $\epsilon e A_{\mu}^{\prime} J_{\mathrm{EM}}^{\mu}$, which involves the electromagnetic current

a e-mail: jtandean@yahoo.com (corresponding author) $e J_{\mathrm{EM}}$ and a small parameter $\epsilon$ due to the kinetic mixing between the dark and SM Abelian gauge fields [1-7]. It follows that $A^{\prime}$ could be produced in the decays or scatterings of SM fermions and hadrons and it might decay into electrically charged fermions or mesons. In general, it could also decay invisibly into other dark particles. These possibilities have stimulated numerous dedicated quests for it, but with negative results so far, leading to bounds on $\epsilon$ over various ranges of the $A^{\prime}$ mass [1-6,26-31].

The massless dark photon, here denoted by $\bar{\gamma}$, is very dissimilar from the massive one because they differ substantially in both theoretical underpinnings and experimental signatures [6-19]. If $\mathrm{U}(1)_{D}$ remains unbroken, one can always arrange a linear combination of the dark and SM U(1) gauge bosons such that it has no renormalizable connection to the SM and can then be identified as the massless dark photon $[7,9]$. Since it therefore does not interact directly with SM members, the limitations implied by the aforementioned hunts for $A^{\prime}$ are not applicable to $\bar{\gamma}$. Nevertheless, the latter could still have consequential impact via higher-dimensional operators [9-11], caused by loop diagrams containing new heavy particles, which may translate into detectable effects. This suggests that potentially promising avenues to seek $\bar{\gamma}$ may be available and hence should be explored. Some of them will be put forward below, which may be feasible at ongoing or near-future experiments. Given that the viable parameter space of the massive dark photon continues to shrink with accumulating null outcomes of its searches, it is of great interest to pay increasing attention to the alternate possibility that the dark photon is massless.

In this paper we concern ourselves with flavor-changing neutral current (FCNC) transitions induced by the massless dark photon, $\bar{\gamma}$, having nonrenormalizable interactions with the $d$ and $s$ quarks described by dimension-five operators in the Lagrangian

$\mathcal{L}_{d s \bar{\gamma}}=-\bar{d}\left(\mathbb{C}+\gamma_{5} \mathbb{C}_{5}\right) \sigma^{\mu \nu}{ }_{s} \bar{F}_{\mu \nu}+$ H.c., 
where $\mathbb{C}$ and $\mathbb{C}_{5}$ are constants which have the dimension of inverse mass and can be complex, $\bar{F}_{\mu \nu}=\partial_{\mu} \bar{A}_{\nu}-\partial_{\nu} \bar{A}_{\mu}$ is the field-strength tensor of $\bar{\gamma}$, and $\sigma^{\mu \nu}=i\left[\gamma^{\mu}, \gamma^{\nu}\right] / 2$. In the absence of other particles beyond the SM lighter than the electroweak scale, $\mathcal{L}_{d s \bar{\gamma}}$ could originate from dimensionsix operators which respect the SM gauge group and the unbroken $\mathrm{U}(1)_{D}$. One can express such operators in the form $\mathcal{L}_{\mathrm{NP}}=-\Lambda_{\mathrm{NP}}^{-2}\left(\mathcal{C}_{12} \overline{Q_{1}} \sigma^{\mu v} d_{2}+\mathcal{C}_{21} \overline{Q_{2}} \sigma^{\mu v} d_{1}\right) H \bar{F}_{\mu \nu}+$ H.c. where $\Lambda_{\mathrm{NP}}$ represents an effective heavy mass scale, the dimensionless coefficients $\mathcal{C}_{12,21}$ are generally complex, $Q_{1,2}$ $\left(d_{1,2}\right)$ stand for left-handed quark doublets (right-handed down-type quark singlets) from the first two families, and $H$ designates the SM Higgs doublet [9]. Accordingly $\mathbb{C} \Lambda_{\mathrm{NP}}^{2}=$ $\left(\mathcal{C}_{12}+\mathcal{C}_{21}^{*}\right) v / \sqrt{8}$ and $\mathbb{C}_{5} \Lambda_{\mathrm{NP}}^{2}=\left(\mathcal{C}_{12}-\mathcal{C}_{21}^{*}\right) v / \sqrt{8}$, with $v \simeq 246 \mathrm{GeV}$ being the Higgs vacuum expectation value. Both $\Lambda_{\mathrm{NP}}$ and $\mathcal{C}_{12,21}$ depend on the details of the underlying new physics (NP).

The interactions in $\mathcal{L}_{d s \bar{\gamma}}$ bring about the FCNC decays of hyperons into a lighter baryon plus missing energy carried away by the massless dark photon. In Ref. [15] we have studied such two-body processes and demonstrated that their rates are allowed by present constraints to reach values that are within the sensitivity reach of the ongoing BESIII experiment $[32,33]$. Analogous transitions can take place in the kaon sector. In the case of massive dark photon, $K \rightarrow \pi A^{\prime}$ and $K^{+} \rightarrow \ell^{+} \nu A^{\prime}$ with $\ell=e, \mu$ might be useful in the quests for it [22-26]. In contrast, since $\bar{\gamma}$ is massless and has no renormalizable links to the $\mathrm{SM}$, angular-momentum conservation and gauge invariance forbid $K \rightarrow \pi \bar{\gamma}$, while $K^{+} \rightarrow \ell^{+} v \bar{\gamma}$ would be highly suppressed. Instead, it has been suggested in Ref. [13] that $\mathcal{L}_{d s \bar{\gamma}}$ could be probed with $K^{+} \rightarrow \pi^{+} \pi^{0} \bar{\gamma}$, which might be accessible in the NA62 experiment [34].

It turns out that there are other kaon modes which may provide additional and competitive windows into the same $d s \bar{\gamma}$ couplings. Specifically, here we propose to pursue the neutral-kaon channels $K_{L} \rightarrow \gamma \bar{\gamma}$ and $K_{L} \rightarrow \pi^{0} \gamma \bar{\gamma}$ and the charged one $K^{+} \rightarrow \pi^{+} \gamma \bar{\gamma}$, all of which have an ordinary photon, $\gamma$, among the daughter particles. As we will show, the two $K_{L}$ modes could have rates which may be big enough to be observable in the currently running KOTO experiment [35]. We will also examine $K_{S} \rightarrow \gamma \bar{\gamma}, \pi^{0} \gamma \bar{\gamma}$ and $K_{L, S} \rightarrow$ $\pi^{+} \pi^{-} \bar{\gamma}$ and take another look at $K^{+} \rightarrow \pi^{+} \pi^{0} \bar{\gamma}$.

The remainder of the paper is organized as follows. In Sect. 2 we first deal with the amplitudes for the kaon decays being analyzed and then calculate their rates. In treating the amplitudes, we need the relevant mesonic matrix-elements of the quark bilinears in Eq. (1). To derive them, we utilize the techniques of chiral perturbation theory. In Sect. 3 we evaluate the maximal branching fractions of the kaon modes, taking into account model-independent restrictions on the $d s \bar{\gamma}$ couplings deduced from the available hyperon data. We draw our conclusions in Sect. 4.

\section{Kaon decay amplitudes and rates}

To investigate the influence of $\mathcal{L}_{d s \bar{\gamma}}$ on our processes of interest, we adopt the framework of chiral perturbation theory [36]. In this context, one can obtain the correspondences between operators comprising bilinears of the quark fields $\left(q_{1}, q_{2}, q_{3}\right)=(u, d, s)$ and their hadronic counterparts involving the lightest pseudoscalar-meson fields, which constitute a flavor-SU(3) octet and are collected into

$$
\begin{aligned}
& \Sigma=e^{i \varphi / f}, \\
& \varphi=\sqrt{2}\left(\begin{array}{ccc}
\frac{1}{\sqrt{2}} \pi^{0}+\frac{1}{\sqrt{6}} \eta_{8} & \pi^{+} & K^{+} \\
\pi^{-} & \frac{-1}{\sqrt{2}} \pi^{0}+\frac{1}{\sqrt{6}} \eta_{8} & K^{0} \\
K^{-} & \bar{K}^{0} & \frac{-2}{\sqrt{6}} \eta_{8}
\end{array}\right),
\end{aligned}
$$

where $f$ denotes the meson decay constant. At the leading chiral order, the bosonization of the quark tensor currents in Eq. (1) has been addressed before [37-41]. Explicitly, it is most generally given by [40]

$$
\begin{aligned}
& \bar{q}_{I} \sigma_{\mu \nu}\left(1-\gamma_{5}\right) q_{J} \\
& \quad \Leftrightarrow-i a_{T} f^{2}\left[\left(\mathcal{D}_{\mu} \Sigma \mathcal{D}_{\nu} \Sigma^{\dagger}-\mathcal{D}_{\nu} \Sigma \mathcal{D}_{\mu} \Sigma^{\dagger}\right.\right. \\
& \left.\left.\quad+i \epsilon_{\mu \nu \varrho \varsigma} \mathcal{D}^{\varrho} \Sigma \mathcal{D}^{\varsigma} \Sigma^{\dagger}\right) \Sigma\right]_{J I}+a_{T}^{\prime} f^{2}\left[\Sigma\left(\mathrm{F}_{\mu \nu}^{R}+i \widetilde{\mathrm{F}}_{\mu \nu}^{R}\right)\right. \\
& \left.\quad+\left(\mathrm{F}_{\mu \nu}^{L}+i \widetilde{\mathrm{F}}_{\mu \nu}^{L}\right) \Sigma\right]_{J I}, \\
& \bar{q}_{I} \sigma_{\mu \nu}\left(1+\gamma_{5}\right) q_{J} \\
& \quad \Leftrightarrow-i a_{T} f^{2}\left[\left(\mathcal{D}_{\mu} \Sigma^{\dagger} \mathcal{D}_{\nu} \Sigma-\mathcal{D}_{\nu} \Sigma^{\dagger} \mathcal{D}_{\mu} \Sigma\right.\right. \\
& \left.\left.\quad-i \epsilon_{\mu \nu \varrho S} \mathcal{D}^{\varrho} \Sigma^{\dagger} \mathcal{D}^{\varsigma} \Sigma\right) \Sigma^{\dagger}\right]_{J I}+a_{T}^{\prime} f^{2}\left[\Sigma^{\dagger}\left(\mathrm{F}_{\mu \nu}^{L}-i \widetilde{\mathrm{F}}_{\mu \nu}^{L}\right)\right. \\
& \left.\quad+\left(\mathrm{F}_{\mu \nu}^{R}-i \widetilde{\mathrm{F}}_{\mu \nu}^{R}\right) \Sigma^{\dagger}\right]_{J I},
\end{aligned}
$$

where $a_{T}$ and $a_{T}^{\prime}$ are constants having the dimension of inverse mass and electromagnetic effects are included via ${ }^{1}$

$$
\begin{aligned}
\mathcal{D}_{\mu} \Sigma & =\partial_{\mu} \Sigma-i \mathrm{~F}_{\mu}^{L} \Sigma+i \Sigma \mathrm{F}_{\mu}^{R}, \\
\mathrm{~F}_{\mu}^{L} & =\mathrm{F}_{\mu}^{R}=-e A_{\mu} Q_{q}, \\
\mathrm{~F}_{\mu \nu}^{L} & =\mathrm{F}_{\mu \nu}^{R}=-e F_{\mu \nu} Q_{q}, \\
\widetilde{\mathrm{F}}_{\mu \nu}^{L} & =\widetilde{\mathrm{F}}_{\mu \nu}^{R}=-e \epsilon_{\mu \nu \varrho \varsigma} \partial^{\varrho} A^{\varsigma} Q_{q}, \\
F_{\mu \nu} & =\partial_{\mu} A_{\nu}-\partial_{\nu} A_{\mu}, \\
Q_{q} & =\frac{1}{3} \operatorname{diag}(2,-1,-1),
\end{aligned}
$$

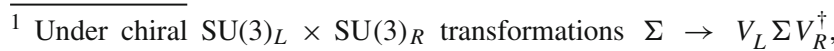
$\mathcal{D}_{\mu} \Sigma \rightarrow V_{L} \mathcal{D}_{\mu} \Sigma V_{R}^{\dagger}, \mathrm{F}_{\mu \nu}^{\chi} \rightarrow V_{\chi} \mathrm{F}_{\mu \nu}^{\chi} V_{\chi}^{\dagger}$, and $\widetilde{\mathrm{F}}_{\mu \nu}^{\chi} \rightarrow V_{\chi} \widetilde{\mathrm{F}}_{\mu \nu}^{\chi} V_{\chi}^{\dagger}$ for $\chi=L, R$, where $V_{\chi} \in \mathrm{SU}(3)_{\chi}$.
} 
with $A_{\mu}$ and $F_{\mu \nu}$ standing for the ordinary photon field and its field-strength tensor, respectively, and $Q_{q}$ representing the electric-charge matrix of the three lightest quarks. Hence the subscript pair $J I=32$ (23) on the right-hand sides of Eq. (3) corresponds to $s \rightarrow d(d \rightarrow s)$ transitions.

This allows us to determine the matrix elements required to write down the amplitudes for $K \rightarrow \gamma \bar{\gamma}$ and $K \rightarrow \pi \gamma \bar{\gamma}$ arising from $\mathcal{L}_{d s \bar{\gamma}}$ which have both an ordinary photon, $\gamma$, and a massless dark photon in the final states. Thus, for $K \rightarrow \gamma \bar{\gamma}$ we arrive at

$$
\begin{aligned}
& \left\langle\gamma\left|\bar{d} \sigma_{\alpha \omega} s\right| \bar{K}^{0}\right\rangle \\
& \quad=\left\langle\gamma\left|\bar{s} \sigma_{\alpha \omega} d\right| K^{0}\right\rangle=\frac{i \sqrt{8}}{3} a_{T}^{\prime} \text { ef } \epsilon_{\alpha \omega \mu \nu} \varepsilon^{* \mu} \mathrm{k}^{\nu}, \\
& \left\langle\gamma\left|\bar{d} \sigma_{\alpha \omega} \gamma_{5} s\right| \bar{K}^{0}\right\rangle \\
& \quad=\left\langle\gamma\left|\bar{s} \sigma_{\alpha \omega} \gamma_{5} d\right| K^{0}\right\rangle=\frac{\sqrt{8}}{3} a_{T}^{\prime} \text { ef }\left(\varepsilon_{\omega}^{*} \mathrm{k}_{\alpha}-\varepsilon_{\alpha}^{*} \mathrm{k}_{\omega}\right),
\end{aligned}
$$

where $\varepsilon$ and $\mathrm{k}$ are the ordinary photon's polarization vector and momentum, respectively. Contracting these matrix elements with the dark photon's polarization vector $\bar{\varepsilon}$ and momentum $\bar{q}$ as dictated by Eq.(1), in conjunction with applying the approximation $\sqrt{2} K_{L}=K^{0}+\bar{K}^{0}$, then yields the amplitude

$$
\begin{aligned}
\mathcal{M}_{K_{L} \rightarrow \gamma \bar{\gamma}}= & \frac{4 a_{T}^{\prime} e f}{3}\left[-\epsilon^{\mu \nu \varrho \varsigma} \operatorname{Re} \mathbb{C}\right. \\
& \left.+\left(g^{\mu \varsigma} g^{\nu \varrho}-g^{\mu \nu} g^{\varrho \varsigma}\right) \operatorname{Im} \mathbb{C}_{5}\right] \varepsilon_{\mu}^{*} \bar{\varepsilon}_{v}^{*} k_{\varrho} \bar{q}_{\zeta} .
\end{aligned}
$$

This leads to the decay rate

$$
\Gamma_{K_{L} \rightarrow \gamma \bar{\gamma}}=\frac{8 \alpha_{\mathrm{e}}}{9}\left(a_{T}^{\prime} f\right)^{2} m_{K^{0}}^{3}\left(|\operatorname{Re} \mathbb{C}|^{2}+\left|\operatorname{Im} \mathbb{C}_{5}\right|^{2}\right),
$$

where $\alpha_{\mathrm{e}}=e^{2} /(4 \pi)=1 / 137$. With $\sqrt{2} K_{S}=K^{0}-\bar{K}^{0}$, the amplitude for $K_{S} \rightarrow \gamma \bar{\gamma}$ and its rate are obtainable from Eqs. (6) and (7), respectively, by making the replacements $\operatorname{Re} \mathbb{C} \rightarrow-i \operatorname{Im} \mathbb{C}$ and $\operatorname{Im} \mathbb{C}_{5} \rightarrow i \operatorname{Re} \mathbb{C}_{5}$.

Similarly, for $K \rightarrow \pi \gamma \bar{\gamma}$ we find

$$
\begin{aligned}
\left\langle\pi^{0} \gamma\left|\bar{d} \sigma_{\alpha \omega} s\right| \bar{K}^{0}\right\rangle & =\left\langle\pi^{0} \gamma\left|\bar{s} \sigma_{\alpha \omega} d\right| K^{0}\right\rangle \\
& =\frac{i \sqrt{2} a_{T}^{\prime} e}{3}\left(\varepsilon_{\omega}^{*} \mathrm{k}_{\alpha}-\varepsilon_{\alpha}^{*} \mathrm{k}_{\omega}\right) \\
\left\langle\pi^{0} \gamma\left|\bar{d} \sigma_{\alpha \omega} \gamma_{5} s\right| \bar{K}^{0}\right\rangle & =\left\langle\pi^{0} \gamma\left|\bar{s} \sigma_{\alpha \omega} \gamma_{5} d\right| K^{0}\right\rangle \\
& =\frac{\sqrt{2} a_{T}^{\prime} e}{3} \epsilon_{\alpha \omega \mu \nu} \varepsilon^{* \nu} \mathrm{k}^{\mu} \\
\left\langle\pi^{-} \gamma\left|\bar{d} \sigma_{\alpha \omega} s\right| K^{-}\right\rangle & =2 i a_{T} e\left[\varepsilon_{\alpha}^{*}\left(p_{K}-p_{\pi}\right)_{\omega}\right. \\
& \left.-\varepsilon_{\omega}^{*}\left(p_{K}-p_{\pi}\right)_{\alpha}\right]
\end{aligned}
$$

$$
\begin{aligned}
& +\frac{2 i a_{T}^{\prime} e}{3}\left(\varepsilon_{\alpha}^{*} \mathrm{k}_{\omega}-\varepsilon_{\omega}^{*} \mathrm{k}_{\alpha}\right), \\
\left\langle\pi^{-} \gamma\left|\bar{d} \sigma_{\alpha \omega} \gamma_{5} s\right| K^{-}\right\rangle= & 2 e \epsilon_{\alpha \omega \mu \nu} \varepsilon^{* \mu} \\
& \times\left[a_{T}\left(p_{K}^{v}-p_{\pi}^{v}\right)+\frac{a_{T}^{\prime}}{3} k^{\nu}\right],
\end{aligned}
$$

where $p_{K}$ and $p_{\pi}$ denote the momenta of the kaon and pion, respectively. From these, we derive the amplitudes for the $K_{L}$ and $K^{-}$modes to be

$$
\begin{aligned}
\mathcal{M}_{K_{L} \rightarrow \pi^{0} \gamma \bar{\gamma}}= & \frac{4 a_{T}^{\prime} e}{3}\left[-\left(g^{\mu \nu} g^{\varrho \zeta}-g^{\mu \varsigma} g^{\nu \varrho}\right) \operatorname{Re} \mathbb{C}\right. \\
& \left.+\epsilon^{\mu \nu \varrho \zeta} \operatorname{Im} \mathbb{C}_{5}\right] \varepsilon_{\mu}^{*} \bar{\varepsilon}_{v}^{*} \mathrm{k}_{\varrho} \bar{q}_{\zeta}, \\
\mathcal{M}_{K^{-} \rightarrow \pi^{-} \gamma \bar{\gamma}}= & 4\left(a_{T}+\frac{a_{T}^{\prime}}{3}\right) e\left[\left(g^{\mu \nu} g^{\varrho \zeta}-g^{\mu \varsigma} g^{\nu \varrho}\right) \mathbb{C}\right. \\
& \left.+i \epsilon^{\mu \nu \varrho \zeta} \mathbb{C}_{5}\right] \varepsilon_{\mu}^{*} \bar{\varepsilon}_{v}^{*} \mathrm{k}_{\varrho} \bar{q}_{\zeta} .
\end{aligned}
$$

They translate into the differential rates

$$
\begin{aligned}
\frac{d \Gamma_{K_{L} \rightarrow \pi^{0} \gamma \bar{\gamma}}}{d s_{\gamma \bar{\gamma}}}= & \frac{\alpha_{\mathrm{e}} a_{T}^{\prime 2} s_{\gamma \bar{\gamma}}^{2}}{72 \pi^{2} m_{K^{0}}^{3}} \mathcal{K}^{\frac{1}{2}}\left(m_{K^{0}}^{2}, m_{\pi^{0}}^{2}, s_{\gamma \bar{\gamma}}\right)\left(|\operatorname{Re} \mathbb{C}|^{2}\right. \\
& \left.+\left|\operatorname{Im} \mathbb{C}_{5}\right|^{2}\right), \\
\frac{d \Gamma_{K^{-} \rightarrow \pi^{-} \gamma \bar{\gamma}}}{d s_{\gamma \bar{\gamma}}}= & \frac{\alpha_{\mathrm{e}}\left(3 a_{T}+a_{T}^{\prime}\right)^{2} s_{\gamma \bar{\gamma}}^{2}}{72 \pi^{2} m_{K^{-}}^{3}} \mathcal{K}^{\frac{1}{2}}\left(m_{K^{-}}^{2}, m_{\pi^{-}}^{2}, s_{\gamma \bar{\gamma}}\right) \\
& \times\left(|\mathbb{C}|^{2}+\left|\mathbb{C}_{5}\right|^{2}\right),
\end{aligned}
$$

where $s_{\gamma \bar{\gamma}}$ stands for the invariant mass squared of the $\gamma \bar{\gamma}$ pair and $\mathcal{K}(x, y, z)=(x-y-z)^{2}-4 y z$. To get each of the corresponding decay rates, the integration range is $0 \leq s_{\gamma \bar{\gamma}} \leq\left(m_{K}-m_{\pi}\right)^{2}$. As in the $K \rightarrow \gamma \bar{\gamma}$ case, the amplitude for $K_{S} \rightarrow \pi^{0} \gamma \bar{\gamma}$ and its differential rate have the same expressions as their $K_{L}$ counterparts in Eqs. (9) and (10), respectively, except that $\operatorname{Re} \mathbb{C}\left(\operatorname{Im} \mathbb{C}_{5}\right)$ is changed to $-i \operatorname{Im} \mathbb{C}\left(i \operatorname{Re} \mathbb{C}_{5}\right)$.

From the $a_{T}$ terms in Eq. (3), we can additionally extract mesonic matrix elements pertaining to processes induced by $\mathcal{L}_{d s \bar{\gamma}}$ without the ordinary photon. Particularly, for the threebody channels $K \rightarrow \pi \pi^{\prime} \bar{\gamma}$ we obtain

$$
\begin{aligned}
& \left\langle\pi^{+}(p) \pi^{-}(\bar{p})\left|\bar{d} \sigma_{\alpha \omega} s\right| \bar{K}^{0}\right\rangle \\
& \quad=\frac{i \sqrt{2} a_{T}}{f} \epsilon_{\alpha \omega \mu \nu}\left(2 \bar{p}^{\mu}+\bar{q}^{\mu}\right) p^{\nu}, \\
& \left\langle\pi^{+}(p) \pi^{-}(\bar{p})\left|\bar{d} \sigma_{\alpha \omega} \gamma_{5} s\right| \bar{K}^{0}\right\rangle \\
& \quad=\frac{\sqrt{2} a_{T}}{f}\left[p_{\alpha}(2 \bar{p}+\bar{q})_{\omega}-p_{\omega}(2 \bar{p}+\bar{q})_{\alpha}\right],
\end{aligned}
$$




$$
\begin{aligned}
& \left\langle\pi^{+}(p) \pi^{-}(\bar{p})\left|\bar{s} \sigma_{\alpha \omega} d\right| K^{0}\right\rangle \\
& \quad=\frac{i \sqrt{2} a_{T}}{f} \epsilon_{\alpha \omega \mu \nu} \bar{p}^{\mu}\left(2 p^{\nu}+\bar{q}^{\nu}\right), \\
& \left\langle\pi^{+}(p) \pi^{-}(\bar{p})\left|\bar{s} \sigma_{\alpha \omega} \gamma_{5} d\right| K^{0}\right\rangle \\
& \quad=\frac{\sqrt{2} a_{T}}{f}\left[(2 p+\bar{q})_{\alpha} \bar{p}_{\omega}-(2 p+\bar{q})_{\omega} \bar{p}_{\alpha}\right], \\
& \left\langle\pi^{0}(p) \pi^{-}(\bar{p})\left|\bar{d} \sigma_{\alpha \omega} s\right| K^{-}\right\rangle \\
& \quad=\frac{i a_{T}}{f} \epsilon_{\alpha \omega \mu \nu}\left[4 \bar{p}^{\mu} p^{\nu}+\left(\bar{p}^{\mu}-p^{\mu}\right) \bar{q}^{\nu}\right], \\
& \left\langle\pi^{0}(p) \pi^{-}(\bar{p})\left|\bar{d} \sigma_{\alpha \omega} \gamma_{5} s\right| K^{-}\right\rangle \\
& \quad=\frac{a_{T}}{f}\left[4 p_{\alpha} \bar{p}_{\omega}-4 p_{\omega} \bar{p}_{\alpha}+(p-\bar{p})_{\alpha} \bar{q}_{\omega}-(p-\bar{p})_{\omega} \bar{q}_{\alpha}\right],
\end{aligned}
$$

where we have applied the relation $p_{K}=p+\bar{p}+\bar{q}$. These lead to the $K_{L}$ and $K^{-}$decay amplitudes, which can be written as

$$
\begin{aligned}
\mathcal{M}_{K_{L} \rightarrow \pi^{+} \pi^{-} \bar{\gamma}} & =\frac{8 a_{T}}{f}\left[\epsilon_{\alpha \omega \mu \nu} \bar{\varepsilon}^{\alpha *} p_{-}^{\omega} p_{+}^{\mu} \bar{q}^{\nu} \operatorname{Re} \mathbb{C}\right. \\
+ & \left.\left(p_{+}^{\mu} p_{-}^{v}-p_{+}^{v} p_{-}^{\mu}\right) \bar{\varepsilon}_{\mu}^{*} \bar{q}_{\nu} \operatorname{Im} \mathbb{C}_{5}\right], \\
\mathcal{M}_{K^{-} \rightarrow \pi^{-} \pi^{0} \bar{\gamma}}= & \frac{8 a_{T}}{f}\left[\epsilon_{\alpha \omega \mu \nu} \bar{\varepsilon}^{\alpha *} p_{-}^{\omega} p_{0}^{\mu} \bar{q}^{v} \mathbb{C}\right. \\
& \left.+i\left(p_{-}^{\mu} p_{0}^{\nu}-p_{-}^{\nu} p_{0}^{\mu}\right) \bar{\varepsilon}_{\mu}^{*} \bar{q}_{\nu} \mathbb{C}_{5}\right],
\end{aligned}
$$

where $p_{+,-, 0}$ represent the momenta of $\pi^{+,-, 0}$, respectively. ${ }^{2}$ We then arrive at the differential rates

$$
\begin{aligned}
& \frac{d \Gamma_{K_{L} \rightarrow \pi^{+} \pi^{-} \bar{\gamma}}}{d \hat{s}} \\
& \quad=\frac{a_{T}^{2}\left(m_{K^{0}}^{2}-\hat{s}\right)^{3}}{96 \pi^{3} f^{2} m_{K^{0}}^{3} \sqrt{\hat{s}}}\left(\hat{s}-4 m_{\pi^{-}}^{2}\right)^{3 / 2}\left[(\operatorname{Re} \mathbb{C})^{2}+\left(\operatorname{Im} \mathbb{C}_{5}\right)^{2}\right],
\end{aligned}
$$

$$
\begin{aligned}
& \frac{d \Gamma_{K^{-} \rightarrow \pi^{-} \pi^{0} \bar{\gamma}}}{d \hat{s}} \\
& \quad=\frac{a_{T}^{2}\left(m_{K^{-}}^{2}-\hat{s}\right)^{3}}{96 \pi^{3} f^{2} m_{K^{-}}^{3} \hat{s}^{2}} \mathcal{K}^{\frac{3}{2}}\left(m_{\pi^{-}}^{2}, m_{\pi^{0}}^{2}, \hat{s}\right)\left(|\mathbb{C}|^{2}+\left|\mathbb{C}_{5}\right|^{2}\right),
\end{aligned}
$$

\footnotetext{
$\left.\overline{{ }^{2} \text { Although }\left\langle\pi^{0}\right.} \pi^{0}\left|\bar{d} \sigma_{\alpha \omega}\left(1, \gamma_{5}\right) s\right| \bar{K}^{0}\right\rangle$ and $\left\langle\pi^{0} \pi^{0}\left|\bar{s} \sigma_{\alpha \omega}\left(1, \gamma_{5}\right) d\right| K^{0}\right\rangle$ from Eq. (3) are not zero, their contributions to the $K_{L, S} \rightarrow \pi^{0} \pi^{0} \bar{\gamma}$ amplitudes vanish. This is consistent with angular momentum conservation and gauge invariance (Bose symmetry) forbidding the pion pair in these decays from having an angular momentum $J_{\pi \pi}=0$ (1), similarly to the $K_{L, S} \rightarrow \pi^{0} \pi^{0} \gamma$ case with the ordinary photon [42]. We can therefore neglect $K_{L, S} \rightarrow \pi^{0} \pi^{0} \bar{\gamma}$, which are chirally suppressed compared to the $K \rightarrow \pi \pi^{\prime} \bar{\gamma}$ modes we consider in Eq. (14).
}

where $\hat{s}$ designates the invariant mass squared of the pion pair. They are to be integrated over $\left(m_{\pi}+m_{\pi^{\prime}}\right)^{2} \leq \hat{s} \leq m_{K}^{2}$ to yield the decay rates. Like before, $d \Gamma_{K_{S} \rightarrow \pi^{+} \pi^{-}} / d \hat{s}$ has the same formula as that in Eq. (15), but with $\operatorname{Re} \mathbb{C}\left(\operatorname{Im} \mathbb{C}_{5}\right)$ replaced with $\operatorname{Im} \mathbb{C}\left(\operatorname{Re} \mathbb{C}_{5}\right)$.

We remark that in Eqs. (5), (8), and (11)-(13) each matrix element of $\bar{d} \sigma_{\alpha \omega} s$ and its $\bar{d} \sigma_{\alpha \omega} \gamma_{5} s$ counterpart are related due to the identity $2 i \sigma_{\alpha \omega} \gamma_{5}=\epsilon_{\alpha \omega \mu \nu} \sigma^{\mu \nu}$ for $\epsilon_{0123}=1$. Furthermore, the amplitudes in Eqs. (6), (9), and (14) respect electromagnetic and $\mathrm{U}(1)_{D}$ gauge invariance.

\section{Kaon decay predictions}

From the results of the preceding section for the (differential) rates of the kaon decays of interest, we can evaluate their branching fractions in terms of the coefficients $\mathbb{C}$ and $\mathbb{C}_{5}$. For the input parameters, we employ $f=f_{\pi}=$ 92.07(85) $\mathrm{MeV}$ and the measured kaon lifetimes and meson masses from Ref. [43], as well as the lattice QCD estimates $a_{T}=0.658(23) / \mathrm{GeV}$ [44] and $a_{T}^{\prime}=3.3(1.1) / \mathrm{GeV}[41,45]$ at a renormalization scale of $2 \mathrm{GeV}$. Thus, with their central values we get

$$
\begin{aligned}
& \mathcal{B}\left(K_{L} \rightarrow \gamma \bar{\gamma}\right)=5.74 \times 10^{12}\left[(\operatorname{Re} \mathbb{C})^{2}+\left(\operatorname{Im} \mathbb{C}_{5}\right)^{2}\right] \mathrm{GeV}^{2}, \\
& \mathcal{B}\left(K_{S} \rightarrow \gamma \bar{\gamma}\right)=1.00 \times 10^{10}\left[(\operatorname{Im} \mathbb{C})^{2}+\left(\operatorname{Re} \mathbb{C}_{5}\right)^{2}\right] \mathrm{GeV}^{2},
\end{aligned}
$$

$\mathcal{B}\left(K_{L} \rightarrow \pi^{0} \gamma \bar{\gamma}\right)=4.95 \times 10^{9}\left[(\operatorname{Re} \mathbb{C})^{2}+\left(\operatorname{Im} \mathbb{C}_{5}\right)^{2}\right] \mathrm{GeV}^{2}$, $\mathcal{B}\left(K_{S} \rightarrow \pi^{0} \gamma \bar{\gamma}\right)=8.67 \times 10^{6}\left[(\operatorname{Im} \mathbb{C})^{2}+\left(\operatorname{Re} \mathbb{C}_{5}\right)^{2}\right] \mathrm{GeV}^{2}$, $\mathcal{B}\left(K^{-} \rightarrow \pi^{-} \gamma \bar{\gamma}\right)=2.67 \times 10^{9}\left(|\mathbb{C}|^{2}+\left|\mathbb{C}_{5}\right|^{2}\right) \mathrm{GeV}^{2}$,

$\mathcal{B}\left(K_{L} \rightarrow \pi^{+} \pi^{-} \bar{\gamma}\right)=4.67 \times 10^{10}\left[(\operatorname{Re} \mathbb{C})^{2}+\left(\operatorname{Im} \mathbb{C}_{5}\right)^{2}\right] \mathrm{GeV}^{2}$, $\mathcal{B}\left(K_{S} \rightarrow \pi^{+} \pi^{-} \bar{\gamma}\right)=8.18 \times 10^{7}\left[(\operatorname{Im} \mathbb{C})^{2}+\left(\operatorname{Re} \mathbb{C}_{5}\right)^{2}\right] \mathrm{GeV}^{2}$, $\mathcal{B}\left(K^{-} \rightarrow \pi^{-} \pi^{0} \bar{\gamma}\right)=1.12 \times 10^{10}\left(|\mathbb{C}|^{2}+\left|\mathbb{C}_{5}\right|^{2}\right) \mathrm{GeV}^{2}$.

Clearly, the predictions for their upper values would depend on how large $\mathbb{C}$ and $\mathbb{C}_{5}$ might be, subject to the pertinent constraints.

The allowed ranges of these coefficients have recently been explored in the contexts of a couple of new-physics models in Refs. [13,14]. Therein it was pointed out that the most relevant restrictions on the coefficients in these NP scenarios were from the data on kaon mixing, which receives loop contributions involving the same new particles that participate in the loop diagrams responsible for the $d s \bar{\gamma}$ couplings. Subsequently, it was shown in Ref. [15] that these interactions also gave rise to the FCNC decays of hyperons into a lighter baryon plus $\bar{\gamma}$ emitted invisibly and that the less restrained of the models could saturate the limits on the couplings inferred from the existing data on hyperon 
Table 1 The second column exhibits the sums of branching fractions of all the observed decays [43] of the $\Lambda, \Sigma^{+}, \Xi^{0}, \Xi^{-}$, and $\Omega^{-}$hyperons and of the $K_{L}$ and $K_{S}$ mesons. The last column contains the upper limits on the branching fractions of yet-unobserved decays of these hadrons deduced from the numbers in the second column, as explained in the text

\begin{tabular}{lll}
\hline Hadron & $\begin{array}{c}\text { Branching-fraction sum } \\
\text { of observed modes }\end{array}$ & $\begin{array}{c}\text { Upper limit on total branching } \\
\text { fraction of yet unobserved modes }\end{array}$ \\
\hline$\Lambda$ & $1.0006 \pm 0.0071$ & $1.4 \times 10^{-2}$ \\
$\Sigma^{+}$ & $1.0005 \pm 0.0042$ & $8.0 \times 10^{-3}$ \\
$\Xi^{0}$ & $1.00000 \pm 0.00017$ & $3.4 \times 10^{-4}$ \\
$\Xi^{-}$ & $1.00000 \pm 0.00042$ & $8.3 \times 10^{-4}$ \\
$\Omega^{-}$ & $1.006 \pm 0.011$ & $1.6 \times 10^{-2}$ \\
$K_{L}$ & $1.0044 \pm 0.0018$ & $1.8 \times 10^{-3}$ \\
$K_{S}$ & $1.00191 \pm 0.00071$ & $7.1 \times 10^{-4}$ \\
\hline
\end{tabular}

decays [43]. This implies that the current hyperon data can already translate into model-independent restrictions on the $d s \bar{\gamma}$ interactions. The extracted bounds on $\mathbb{C}$ and $\mathbb{C}_{5}$ can then be used to estimate the maximal values of the kaon branching fractions in Eqs. (17)-(19).

To discuss the impact of the hyperon data more quantitatively, we reproduce here the branching fractions of the aforementioned FCNC hyperon modes calculated in Ref. [15] and expressed in terms of $\mathbb{C}$ and $\mathbb{C}_{5}$ :

$$
\begin{aligned}
& \mathcal{B}(\Lambda \rightarrow n \bar{\gamma})=2.75 \times 10^{12}\left(|\mathbb{C}|^{2}+\left|\mathbb{C}_{5}\right|^{2}\right) \mathrm{GeV}^{2}, \\
& \mathcal{B}\left(\Sigma^{+} \rightarrow p \bar{\gamma}\right)=1.54 \times 10^{11}\left(|\mathbb{C}|^{2}+\left|\mathbb{C}_{5}\right|^{2}\right) \mathrm{GeV}^{2}, \\
& \mathcal{B}\left(\Xi^{0} \rightarrow \Lambda \bar{\gamma}, \Sigma^{0} \bar{\gamma}\right)=1.61 \times 10^{12}\left(|\mathbb{C}|^{2}+\left|\mathbb{C}_{5}\right|^{2}\right) \mathrm{GeV}^{2}, \\
& \mathcal{B}\left(\Xi^{-} \rightarrow \Sigma^{-} \bar{\gamma}\right)=1.32 \times 10^{12}\left(|\mathbb{C}|^{2}+\left|\mathbb{C}_{5}\right|^{2}\right) \mathrm{GeV}^{2}, \\
& \mathcal{B}\left(\Omega^{-} \rightarrow \Xi^{-} \bar{\gamma}\right)=5.18 \times 10^{12}\left(|\mathbb{C}|^{2}+\left|\mathbb{C}_{5}\right|^{2}\right) \mathrm{GeV}^{2} .
\end{aligned}
$$

These transitions, if occur, would be among the yet-unobserved decays of the hyperons. The branching fractions of the latter have approximate maxima which we can determine indirectly from the data on the observed channels quoted by the Particle Data Group [43]. To do so, for each of the parent hyperons, we subtract from unity the sum of the PDG branching-fraction numbers with their errors (increased to 2 sigmas) combined in quadrature. We have collected the results in the third column of Table 1, where the second column displays the sums of the branching-fraction values. ${ }^{3}$

Comparing the hyperon entries in the last column of this table with Eq. (20), we see that the $\Xi^{0}$ bound is the most stringent and leads to

$|\mathbb{C}|^{2}+\left|\mathbb{C}_{5}\right|^{2}<\frac{2.1 \times 10^{-16}}{\mathrm{GeV}^{2}}$

\footnotetext{
${ }^{3}$ In obtaining these entries, if the PDG numbers have asymmetric errors, the lower ones are selected.
}

Combining this with Eqs. (17)-(19) and assuming that for the $K_{L}\left(K_{S}\right)$ cases $\operatorname{Im} \mathbb{C}=\mathbb{C}_{5}=0\left(\mathbb{C}=\operatorname{Im} \mathbb{C}_{5}=0\right)$, we then find

$$
\begin{aligned}
\mathcal{B}\left(K_{L} \rightarrow \gamma \bar{\gamma}\right) & <1.2 \times 10^{-3}, \\
\mathcal{B}\left(K_{S} \rightarrow \gamma \bar{\gamma}\right) & <2.1 \times 10^{-6}, \\
\mathcal{B}\left(K_{L} \rightarrow \pi^{0} \gamma \bar{\gamma}\right) & <1.0 \times 10^{-6}, \\
\mathcal{B}\left(K_{S} \rightarrow \pi^{0} \gamma \bar{\gamma}\right) & <1.8 \times 10^{-9}, \\
\mathcal{B}\left(K_{L} \rightarrow \pi^{+} \pi^{-} \bar{\gamma}\right) & <9.8 \times 10^{-6}, \\
\mathcal{B}\left(K_{S} \rightarrow \pi^{+} \pi^{-} \bar{\gamma}\right) & <1.7 \times 10^{-8}, \\
\mathcal{B}\left(K^{-} \rightarrow \pi^{-} \gamma \bar{\gamma}\right) & <5.6 \times 10^{-7}, \\
\mathcal{B}\left(K^{-} \rightarrow \pi^{-} \pi^{0} \bar{\gamma}\right) & <2.4 \times 10^{-6} .
\end{aligned}
$$

It is worth noting that the numbers in the last line for the $K^{-}$decays are equal to their $K^{+}$counterparts. Furthermore, the predictions in Eq. (22) for the modes with an ordinary photon have uncertainties of up to about $70 \%$ because their rates depend on $a_{T}^{\prime}$ which has an error of order $30 \%$.

The second column of Table 1 also lists the sums of the branching fractions of the observed $K_{L, S}$ decay channels. Since the central values of these numbers exceed unity by more than 2 sigmas, we may demand that the upper limits on the branching fractions of yet-unobserved $K_{L, S}$ modes be less than the errors shown in the second column. Evidently, these requirements, which are quoted in the last two rows of the third column of the table, are satisfied by the respective $K_{L, S}$ predictions in Eq. (22).

\section{Conclusions}

To date there have been numerous dedicated hunts for the massive dark photon, but they still have come up empty. If the dark photon exists and turns out to be massless, it would have eluded those quests for the massive one. Therefore, it is 
essential that future attempts to look for dark photons accommodate the possibility that they are massless, in which case they may have nonnegligible FCNC interactions with SM fermions via higher-dimensional operators.

In this study, we have entertained the latter scenario, specifically that in which the massless dark photon has dipole-type flavor-changing couplings to the $d$ and $s$ quarks. Concentrating on the implications for the kaon sector, and taking into account indirect model-independent constraints on the $d s \bar{\gamma}$ interactions inferred from the available hyperon data, we examine especially $K_{L} \rightarrow \gamma \bar{\gamma}$ and $K_{L} \rightarrow \pi^{0} \gamma \bar{\gamma}$, both of which have an ordinary photon in the final states, and demonstrate that their rates can reach levels which are potentially testable by KOTO. Moreover, $K^{+} \rightarrow \pi^{+} \gamma \bar{\gamma}$ and $K^{+} \rightarrow \pi^{+} \pi^{0} \bar{\gamma}$ can have rates which might be big enough to be accessible by NA62. We have previously proposed that the corresponding hyperon decays with missing energy could be probed by BESIII. It follows that one or more of these presently running experiments may soon discover the massless dark photon or, if not, come up with improved restraints on the $d s \bar{\gamma}$ interactions. In any case, the results of this analysis will hopefully help stimulate efforts to seek massless dark photons in ongoing and near-future kaon and hyperon measurements.

Acknowledgements We would like to thank Yu-Chen Tung for experimental information which motivated this research. It was supported in part by the MOST (Grant No. MOST 106-2112-M-002-003-MY3).

Data Availability Statement This manuscript has no associated data or the data will not be deposited. [Authors' comment: This work is theoretical and hence yields no experimental data.]

Open Access This article is licensed under a Creative Commons Attribution 4.0 International License, which permits use, sharing, adaptation, distribution and reproduction in any medium or format, as long as you give appropriate credit to the original author(s) and the source, provide a link to the Creative Commons licence, and indicate if changes were made. The images or other third party material in this article are included in the article's Creative Commons licence, unless indicated otherwise in a credit line to the material. If material is not included in the article's Creative Commons licence and your intended use is not permitted by statutory regulation or exceeds the permitted use, you will need to obtain permission directly from the copyright holder. To view a copy of this licence, visit http://creativecomm ons.org/licenses/by/4.0/.

Funded by SCOAP ${ }^{3}$.

\section{References}

1. J. Jaeckel, A. Ringwald, The low-energy frontier of particle physics. Ann. Rev. Nucl. Part. Sci. 60, 405 (2010). arXiv:1002.0329 [hep-ph]

2. R. Essig et al., Working Group Report: New Light Weakly Coupled Particles. arXiv:1311.0029 [hep-ph]

3. J. Alexander et al., Dark sectors 2016 workshop: community report. arXiv:1608.08632 [hep-ph]
4. J. Beacham et al., Physics beyond colliders at CERN: beyond the standard model working group report. J. Phys. G 47(1), 010501 (2020). arXiv:1901.09966 [hep-ex]

5. S.N. Gninenko, N.V. Krasnikov, V.A. Matveev, Search for dark sector physics with NA64. arXiv:2003.07257 [hep-ph]

6. M. Fabbrichesi, E. Gabrielli, G. Lanfranchi, The dark photon. arXiv:2005.01515 [hep-ph]

7. B. Holdom, Two U(1)'s and $\epsilon$ charge shifts. Phys. Lett. 166B, 196 (1986)

8. F. del Aguila, M. Masip, M. Perez-Victoria, Physical parameters and renormalization of $U(1)^{a} \times U(1)^{b}$ models. Nucl. Phys. B 456, 531 (1995). [hep-ph/9507455]

9. B.A. Dobrescu, Massless gauge bosons other than the photon. Phys. Rev. Lett. 94, 151802 (2005). [hep-ph/0411004]

10. S. Hoffmann, Paraphotons and Axions: Similarities in Stellar Emission and Detection. Phys. Lett. B 193, 117 (1987)

11. E. Gabrielli, B. Mele, M. Raidal, E. Venturini, FCNC decays of standard model fermions into a dark photon. Phys. Rev. D 94(11), 115013 (2016). arXiv:1607.05928 [hep-ph]

12. D. Fargion,M. Khlopov, C.A. Stephan, Cold dark matter by heavy double charged leptons?Class.Quant.Grav. 23, 7305-7354 (2006). arXiv:astro-ph/0511789 [astro-ph]

13. M. Fabbrichesi, E. Gabrielli, B. Mele, Hunting down massless dark photons in Kaon physics. Phys. Rev. Lett. 119(3), 031801 (2017). arXiv:1705.03470 [hep-ph]

14. M. Fabbrichesi, E. Gabrielli, Dark-sector physics in the search for the rare decays $K^{+} \rightarrow \pi^{+} \nu \bar{v}$ and $K_{L} \rightarrow \pi^{0} v \bar{\nu}$. Eur. Phys. J. C 80(6), 532 (2020). arXiv:1911.03755 [hep-ph]

15. J.Y. Su, J. Tandean, Searching for dark photons in hyperon decays. Phys. Rev. D 101(3), 035044 (2020). arXiv:1911.13301 [hep-ph]

16. J.Y. Su, J. Tandean, Seeking massless dark photons in the decays of charmed hadrons. arXiv:2005.05297 [hep-ph]

17. J.X. Pan, M. He, X.G. He, G. Li, Scrutinizing a massless dark photon: basis independence. Nucl. Phys. B 953, 114968 (2020). arXiv:1807.11363 [hep-ph]

18. R. Foot, S. Vagnozzi, Dissipative hidden sector dark matter. Phys. Rev. D 91, 023512 (2015). arXiv: 1409.7174 [hep-ph]

19. R. Foot, S. Vagnozzi, Diurnal modulation signal from dissipative hidden sector dark matter. Phys. Lett. B 748, 61 (2015). arXiv:1412.0762 [hep-ph]

20. R. Foot, X.G. He, Comment on $Z-Z^{\prime}$ mixing in extended gauge theories. Phys. Lett. B 267, 509 (1991)

21. M. He, X.G. He, C.K. Huang, G. Li, Search for a heavy dark photon at future $e^{+} e^{-}$colliders. JHEP 1803, 139 (2018). arXiv:1712.09095 [hep-ph]

22. P. Fayet, Constraints on light dark matter and $\mathrm{U}$ bosons, from $\psi$, $\Upsilon, K^{+}, \pi^{0}, \eta$ and $\eta^{\prime}$ decays. Phys. Rev. D 74, 054034 (2006). [hep-ph/0607318]

23. M. Pospelov, Secluded U(1) below the weak scale. Phys. Rev. D 80, 095002 (2009). arXiv: 0811.1030 [hep-ph]

24. M. Reece, L.T. Wang, Searching for the light dark gauge boson in GeV-scale experiments. JHEP 0907, 051 (2009). arXiv:0904.1743 [hep-ph]

25. C.W. Chiang, P.Y. Tseng, Probing a dark photon using rare leptonic kaon and pion decays. Phys. Lett. B 767, 289 (2017). arXiv: 1612.06985 [hep-ph]

26. J.R. Batley et al., [NA48/2 Collaboration], Search for the dark photon in $\pi^{0}$ decays. Phys. Lett. B 746, 178 (2015). arXiv:1504.00607 [hep-ex]

27. R. Aaij et al., [LHCb Collaboration], Search for dark photons produced in $13 \mathrm{TeV} p p$ collisions. Phys. Rev. Lett. 120(6), 061801 (2018). arXiv: 1710.02867 [hep-ex]

28. A. Anastasi et al., [KLOE-2 Collaboration], Combined limit on the production of a light gauge boson decaying into $\mu^{+} \mu^{-}$and $\pi^{+} \pi^{-}$. Phys. Lett. B 784, 336 (2018). arXiv:1807.02691 [hep-ex] 
29. M. Ablikim et al., [BESIII Collaboration], Measurement of $\mathcal{B}\left(J / \psi \rightarrow \eta^{\prime} e^{+} e^{-}\right)$and search for a dark photon. Phys. Rev. D 99(1), 012013 (2019) arXiv:1809.00635 [hep-ex]

30. E. Cortina Gil et al., [NA62 Collaboration], Search for production of an invisible dark photon in $\pi^{0}$ decays. JHEP 1905, 182 (2019). arXiv:1903.08767 [hep-ex]

31. D. Banerjee et al., Dark matter search in missing energy events with NA64. Phys. Rev. Lett. 123(12), 121801 (2019). arXiv: 1906.00176 [hep-ex]

32. H.B. Li, Prospects for rare and forbidden hyperon decays at BESIII. Front. Phys. (Beijing) 12(5), 121301 (2017). arXiv:1612.01775 [hep-ex] (Erratum) 14, 64001 (2019)

33. M. Ablikim et al., Future physics programme of BESIII. Chin. Phys. C 44(4), 040001 (2020). arXiv:1912.05983 [hep-ex]

34. E. Cortina Gil et al., [NA62 Collaboration], The beam and detector of the NA62 experiment at CERN. JINST 12(05), P05025 (2017). arXiv:1703.08501 [physics.ins-det]

35. J.K. Ahn et al., [KOTO Collaboration], Search for the $K_{L} \rightarrow \pi^{0} v \bar{v}$ and $K_{L} \rightarrow \pi^{0} X^{0}$ decays at the J-PARC KOTO experiment. Phys. Rev. Lett. 122(2), 021802 (2019). arXiv:1810.09655 [hep-ex]

36. J. Gasser, H. Leutwyler, Chiral perturbation theory: expansions in the mass of the strange quark. Nucl. Phys. B 250, 465 (1985)
37. G. Colangelo, G. Isidori, J. Portoles, Supersymmetric contributions to direct CP violation in $K \rightarrow \pi \pi \gamma$ decays. Phys. Lett. B 470, 134 (1999). [hep-ph/9908415]

38. J. Tandean, G. Valencia, Reanalysis of CP violation in $K_{L} \rightarrow$ $\pi^{+} \pi^{-} \gamma$. Phys. Rev. D 62, 116007 (2000). [hep-ph/0008238]

39. D.N. Gao, Charge asymmetry in $K^{+} \rightarrow \pi^{ \pm} \gamma \gamma$ induced by the electromagnetic penguin operators. Phys. Rev. D 67, 074028 (2003). [hep-ph/0212280]

40. P. Mertens, C. Smith, The $s \rightarrow d \gamma$ decay in and beyond the standard model. JHEP 1108, 069 (2011). arXiv:1103.5992 [hep-ph]

41. J.F. Kamenik, C. Smith, FCNC portals to the dark sector. JHEP 1203, 090 (2012). arXiv:1111.6402 [hep-ph]

42. T.D. Lee, C.S. Wu, Weak interactions: decays of neutral K mesons. Ann. Rev. Nucl. Part. Sci. 16, 511 (1966)

43. M. Tanabashi et al., [Particle Data Group], Review of particle physics. Phys. Rev. D 98(3), 030001 (2018)

44. I. Baum, V. Lubicz, G. Martinelli, L. Orifici, S. Simula, Matrix elements of the electromagnetic operator between kaon and pion states. Phys. Rev. D 84, 074503 (2011). arXiv:1108.1021 [hep-lat]

45. P.V. Buividovich, M.N. Chernodub, E.V. Luschevskaya, M.I. Polikarpov, Lattice QCD in strong magnetic fields. eCONF C 0906083, 25 (2009). arXiv:0909.1808 [hep-ph] 